\title{
Circulating magnesium levels and incidence of coronary heart diseases, hypertension, and type 2 diabetes mellitus: a meta-analysis of prospective cohort studies
}

\author{
Jiang $\mathrm{Wu}^{1,2,3 \dagger}$, Pengcheng $\mathrm{Xun}^{4 \dagger}{ }^{4 \dagger}$ Qingya Tang ${ }^{1,2,3}$, Wei Cai ${ }^{1,2,3,5^{*}}$ and $\mathrm{Ka} \mathrm{He}{ }^{4^{*}}$
}

\begin{abstract}
Background: Data on the associations between circulating magnesium (Mg) levels and incidence of coronary heart diseases (CHD), hypertension, and type 2 diabetes mellitus (T2DM) are inconsistent and inconclusive. The aim of this study was to examine circulating Mg levels in relation to incidence of CHD, hypertension, and T2DM.

Methods: Prospective cohort studies published before May 2017 were searched through PubMed, EmBase, SCOPUS, and Google Scholar. A total of 11 studies that reported multivariable-adjusted associations of interest were identified. Information on the characteristics of study and participants, exposure, main outcomes, risk estimates, and cofounders was extracted and analyzed.

Results: Of the 11 included studies, 5 reported results on CHD (38,808 individuals [4437 cases] with an average 10.5year follow-up), 3 on hypertension (14,876 participants [3149 cases] with a 6.7-year follow-up), and 4 on T2DM (31,284 participants [2680 cases] with an 8.8-year follow-up). Comparing the highest to the lowest category of circulating Mg concentration, the pooled relative risks [RRs] (95\% confidence intervals [Cls]) were $0.86(0.74,0.996), 0.91(0.80,1.02)$, and $0.64(0.50,0.81)$ for incidence of CHD, hypertension, and T2DM, respectively. Every $0.1 \mathrm{mmol} / \mathrm{L}$ increment in circulating Mg levels was associated with 4\% (RR, 0.96; 95\% Cl: 0.94, 0.99) reduction in hypertension incidence. No significant linear association was found between circulating Mg levels and incidence of CHD (RR, $0.89 ; 95 \% \mathrm{Cl}: 0.77,1.03)$ and T2DM (RR, 0.90; 95\% Cl: 0.81, 1.002). The observed associations of interest were sensitive to exclusion of individual studies.

Conclusions: Findings in this meta-analysis suggest that circulating Mg levels are inversely associated with incidence of $\mathrm{CHD}$, hypertension, and T2DM. Additional studies are needed to provide more solid evidence and identify the optimal range of circulating $\mathrm{Mg}$ concentration with respect to primary prevention of CHD, hypertension, and T2DM.
\end{abstract}

Keywords: Magnesium, Coronary heart disease, Hypertension, Type 2 diabetes, Meta-analysis

\footnotetext{
* Correspondence: caiw1978@163.com; kahe@indiana.edu

Jiang Wu and Pengcheng Xun are co-first authors of this paper.

${ }^{\dagger}$ Equal contributors

'Department of Clinical Nutrition, Xin Hua Hospital, School of Medicine,

Shanghai Jiao Tong University, No. 1665, Kongjiang Rd, Shanghai, China

${ }^{4}$ Department of Epidemiology and Biostatistics, School of Public

Health-Bloomington, Indiana University, 1025 E. Seventh Street, C042,

Bloomington, Indiana 47405, USA

Full list of author information is available at the end of the article
} 


\section{Introduction}

Studies suggest that coronary heart diseases (CHD), hypertension, and type 2 diabetes mellitus (T2DM) are comorbidities and the major risk factors of mortality $[1,2]$. Incidences of these chronic diseases are growing rapidly [3-5], and identifying modifiable risk factors is crucial to the prevention of these diseases.

Magnesium ( $\mathrm{Mg}$ ) is the second most predominant intracellular electrolyte, following after potassium. Mg serves as an important cofactor in many essential enzymatic reactions involved in glucose metabolism and several essential physiological processes, including modulating vascular smooth muscle tone and endothelial cell function [6-8]. The role of $\mathrm{Mg}$ in the development and progress of T2DM and cardiovascular diseases (CVD) has drawn researchers' attention in the recent decades [9-13]. Several large prospective cohort studies have shown that low $\mathrm{Mg}$ intake is associated with incidence of T2DM and CVD [11, 12, 1416]. Notably, because Mg intake was usually assessed based on self-administered food frequency questionnaires $[11,15$, 16], misclassification is inevitable. In addition, dietary $\mathrm{Mg}$ could not represent the accurate amount of $\mathrm{Mg}$ intake without considering the substantial loss during food processing and cooking [17]. Moreover, the health impact of $\mathrm{Mg}$ is difficult to be distinguished from intake of other nutrients such as calcium, potassium, phosphorus, and fiber.

Serum or plasma $\mathrm{Mg}$ concentration is the most commonly used biomarker to assess $\mathrm{Mg}$ metabolism abnormality in clinical practice. It reflects not only the dietary intake, but also the intestinal absorption, renal reabsorption and excretion, and hormone regulation. Healthy individuals maintain quite stable circulating $\mathrm{Mg}$ levels, except for in cases of $\mathrm{Mg}$ deficiency. Of note, the preponderance of epidemiological and clinical information relevant to chronic disease associations with $\mathrm{Mg}$ status is derived from serum total Mg levels, which reasonably approximates ionized (free) Mg concentration [18]. Other biomarkers such as 24$\mathrm{h}$ urine $\mathrm{Mg}$, red blood cell $\mathrm{Mg}$, and ionized $\mathrm{Mg}$ were often restricted in large-scale epidemiological studies due to budget and ethical considerations. A recently published meta-analysis on randomized controlled trials revealed a dose-response relationship of oral $\mathrm{Mg}$ supplementation with circulating Mg levels [19], which supports circulating $\mathrm{Mg}$ as a reasonable biomarker of $\mathrm{Mg}$ status.

Many studies suggest that low circulating Mg levels are associated with insulin resistance $[12,20,21]$, which is a known risk factor of T2DM, CHD and hypertension [22]. A number of prospective cohort studies have examined the association between circulating $\mathrm{Mg}$ levels and incidence of CHD [23-27], hypertension [26, 28, 29], or T2DM [11, 30-32], but the findings are inconsistent and inconclusive. One recent meta-analysis examined both dietary intake of $\mathrm{Mg}$ and circulating $\mathrm{Mg}$ levels with the risk of CHD found an inverse association [33]. Earlier, two meta-analyses found that circulating $\mathrm{Mg}$ concentrations were inversely related to the risk of CVD [13, 34]. However, dietary intake of $\mathrm{Mg}$ is subject to measurement error. Also, CVD includes a number of outcomes that may have different pathophysiology, e.g., ischemic vs. hemorrhagic stroke. In addition, studies suggest that CHD, hypertension, and T2DM are comorbidities and the major risk factors of mortality. Of note, these three chronic diseases share the same risk factor - insulin resistance, which is closely related to circulating $\mathrm{Mg}$ levels. Therefore, we aimed to quantitatively summarize the literature by conducting a meta-analysis of prospective cohort studies on the associations of circulating $\mathrm{Mg}$ levels with incidence of CHD, hypertension, and T2DM.

\section{Methods}

This meta-analysis was conducted following the preferred reporting items for systematic reviews and metaanalyses (PRISMA) guideline [35]. The completed PRISMA checklist is available in Additional file 1: Table S1 (see supplemental materials).

\section{Data sources and search strategy}

We conducted a systematic literature review to identify all the studies on the associations between circulating $\mathrm{Mg}$ levels and incidence of CHD, hypertension, or T2DM through May 2017. We first searched the electronic database of PubMed (https://www.ncbi.nlm.nih.gov/pubmed) using the following MeSH terms: ("micronutrients" OR "magnesium" OR "magnesium deficiency") AND ("cardiovascular disease" OR "myocardial infarction" OR "ischemic heart disease" OR "hypertension" OR "blood pressure" OR "coronary heart disease" OR "diabetes mellitus" OR "hyperglycemia" OR "insulin resistance") AND ("cohort studies" or "follow-up studies" OR "longitudinal studies" OR "prospective studies"). We further reviewed EmBase (http://www.elsevier.com/online-tools/embase), SCOPUS (https://www.scopus.com/) and Google Scholar (http://scholar.google.com/). In addition, we identified relevant articles by manually searching the references of the retrieved studies and review articles.

\section{Study selection}

Articles were selected if they met the following criteria: published in English; had a prospective design; evaluated the association between baseline circulating $\mathrm{Mg}$ levels and CHD, hypertension, or T2DM; and reported estimated relative risks (RRs), hazard ratios (HRs), or odds ratios (ORs) with 95\% confidence intervals (CIs) or these data could be calculated from the available information. In the included studies, CHD was defined as any coronary heart disease, including ischemic heart disease (IHD) incidence or death, angina, myocardial infarction and sudden cardiac death. Hypertension was defined as a systolic blood 
pressure $\geq 140 \mathrm{mmHg}$, or a diastolic blood pressure $\geq 90 \mathrm{mmHg}$, or the use of anti-hypertensive drugs. T2DM was defined based on the blood glucose levels (fasting plasma glucose $\geq 7.0 \mathrm{mmol} / \mathrm{l}$, or, non-fasting or $2 \mathrm{~h}$ post-load glucose $\geq 11.1 \mathrm{mmol} / \mathrm{l}$ ) or the use of antidiabetic drugs. New-onset CHD, hypertension and T2DM was determined by medical records, self-reports or death certificates. All the articles were identified by an initial screen of abstracts, followed by a full-text review, which was independently conducted by two investigators (JW and PX).

\section{Data extraction and quality assessment}

The relevant data were independently extracted by two investigators (JW and PX) using a standardized form, and disagreements were resolved by consensus after discussion with the third investigator $(\mathrm{KH})$. The following information was extracted: study characteristics (study name, publication year, authors, country where the study was conducted, study design, sample size, and follow-up time), participants' characteristics (age at baseline, race, proportion of male gender, major covariates), exposure (method of assessment, classification), main outcomes, and estimated RRs with 95\% CIs for corresponding categories and/or continuous exposure. If more than one multivariate model was reported, the estimates with full adjustment for potential confounders were extracted.

The quality of included studies was ascertained with the Newcastle-Ottawa quality assessment scale (NOS) [36] by 2 investigators (JW and PX) independently. Any disagreement was solved by group discussion with the third investigator $(\mathrm{KH})$. This assessment allowed a total score of up to 9 points. The NOS for cohort studies was divided into three groups: selection of cohort (4 points), comparability of cohort (2 points), and assessment of outcome (3 points). The quality of study was considered high or moderate if the sum score was $\geq 8$ points or between 5 and 7 points, respectively.

\section{Statistical methods}

All analyses were performed by using STATA statistical software (Version 13.0; STATA Corporation LP, College Station, Texas, US). Unless otherwise specified, a value of $P \leq 0.05$ was considered statistically significant.

In this meta-analysis, we used RRs and 95\% CIs as a measure of the effect size for all studies. Hazards ratio was considered RR directly, and OR was considered RR in the main analysis and was transformed into $R R$ in a sensitivity analysis by using the formula: $\mathrm{RR}=\mathrm{OR} /[(1-$ $\left.\left.\left.P_{0}\right)+\mathrm{OR}^{*} P_{0}\right)\right]$, where $P_{0}$ indicated incidence of the outcome of interest in the reference group [37]. RRs and 95\% CIs transformed to their natural logarithms (ln) were used to compute the corresponding standard errors. RRs and 95\% CIs were converted to per $0.1 \mathrm{mmol} /$
$\mathrm{L}$ increment consistently to describe the linear associations of interest regardless of the original unit $(\mathrm{mEq} / \mathrm{L}$, $\mathrm{mmol} / \mathrm{L}$ or $\mathrm{mg} / \mathrm{dL}$ ) for $\mathrm{Mg}$ levels. If a study did not provide the linear association of circulating $\mathrm{Mg}$ concentration with the outcomes of interest, we estimated it based on the categorical associations by using Greenland and Longnecker's method, if the person-time of participants as well as cases were reported for each subgroup of $\mathrm{Mg}$ levels [38], or using variance-weighted least squares linear regression if they were not reported. If the highest or the lowest group of $\mathrm{Mg}$ levels was an open range, then its upper or lower limit was estimated by assuming its range as the same width as the adjacent category.

We pooled RR estimates separately for each outcome using a random-effects model. We evaluated the statistical heterogeneity of the RRs using Cochran's $Q$ test with a significance level of 0.10 , and quantified the heterogeneity using the $I^{2}$ statistic with a value of $0-25 \%$, $26-50 \%, 51-75 \%$, or $>75 \%$ denoting very low, low, moderate, and high degrees of heterogeneity, respectively. Publication bias was visually examined by funnel plots, and statistically assessed by using Egger's regression asymmetry test and Begg's rank correlation test, with a significance level of 0.10 . If publication bias existed, the Duval and Tweedie nonparametric "trim and fill" method was used to adjust for the pooled results [39].

The average of follow-up year was calculated as the sum of person-year divided by total number of participants. Sensitivity analyses were conducted to evaluate the influence of replacing the random-effects model with the fixed-effects model and the influence of a single study on the overall association by calculating the pooled estimates while omitting one primary study at each time. In addition, OR was transformed to RR in a sensitivity analysis.

\section{Results \\ Literature search}

As shown in Fig. 1, we retrieved 805 relevant articles from PubMed. Of them, 795 articles were excluded for one of the following reasons: 1) not a human study; 2) a review/ meta-analysis, editorial, or abstract; 3) not published in English; 4) not conducted in the general population, but in patients (e.g., diabetic patients); 5) not relating circulating $\mathrm{Mg}$ levels to an outcome of interest; 6) not a prospective cohort design; or 7) did not exclude prevalent cases at baseline. In addition, we found 1 additional articles by searching Google Scholar. Thus, 11 studies were identified and included in this meta-analysis.

\section{Characteristics of included studies}

Table 1 summarizes the characteristics of the included studies, all of which had a prospective cohort design and had participants without a prior diagnosed outcome of 


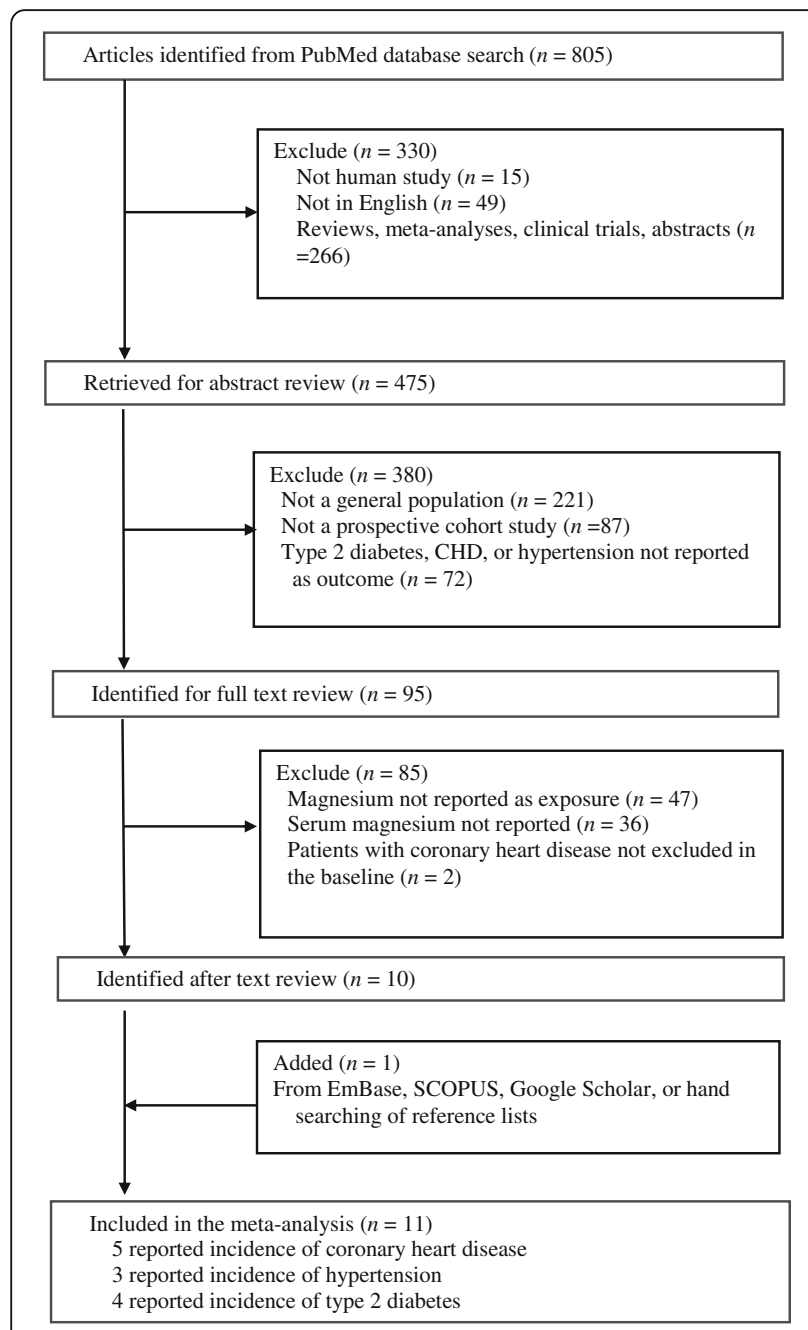

Fig. 1 Study selection process. Articles were identified by searches of PubMed (http://www.ncbi.nlm.nih.gov/pubmed), EmBase (http:// www.elsevier.com/online-tools/embase), SCOPUS (https://

www.scopus.com/) and Google Scholar (http://scholar.google.com)

interest at baseline. Some studies estimated RRs/HRs/ORs with 95\% CIs for men and women separately [24, 28], or for blacks and whites independently [11], and these were counted as separate cohorts in the meta-analysis. Of these 11 prospective studies, 5 studies (6 cohorts) reported results on CHD with 45,808 individuals and 4437 cases during an average of 10.5 years of follow-up [23-27], 3 studies (4 cohorts) on hypertension with 14,876 participants and 3149 cases during an average of 6.7 years of follow-up [26, 28, 29 ], and 4 studies (5 cohorts) on T2DM with 31,284 participants and 2680 cases during an average of 8.8 years of follow-up [11, 30-32].

The average age at baseline was approximately 52 years old, and about $43.4 \%$ participants were male. All the included studies adjusted for age and sex, except for the studies that only included one gender. Also, the primary studies controlled for various potential confounders, including body mass index $(n=9)[11,23,25-30,32]$ and/or other body composition variables such as waist/hip ratio $(n=3)$ [11, 24,28 ] and waist circumference $(n=1)$ [31], smoking $(n=7)[23-27,29,32]$, alcohol consumption $(n=7)$ $[11,23-25,27,29,32]$, physical activity $(n=6)[11$, $23-25,28,30]$, and education $(n=5) \quad[11,24,25,28$, 30]. Few studies adjusted for circulating levels of other nutrients such as calcium and potassium $(n=4)[11,27,29,32]$ or estimated glomerular filtration rate $(n=2)[26,32]$. Ten out of the 11 included studies were assessed as high quality and only 1 yielded moderate quality (see Additional file 1: Table S2 in supplemental materials).

\section{Circulating magnesium levels and CHD risk}

Five studies (6 cohorts) reported data for CHD (Fig. 2). The summary estimate was 0.86 (95\% CI: 0.74, 0.996; $P=0.04)$. There was no significant heterogeneity among the studies $\left(I^{2}=39.6 \%, P=0.14\right)$ and no significant publication bias (Egger's test: $P=0.48$; Begg's test: $P=0.57$ ).

Dose-response analysis did not reveal a linear association between circulating $\mathrm{Mg}$ levels and incidence of CHD (RR, 0.89; $95 \%$ CI: $0.77,1.03$; $P_{\text {trend }}=0.10$ with a $0.1 \mathrm{mmol} / \mathrm{L}$ increment in $\mathrm{Mg}$ levels). High heterogeneity was observed among the 6 included cohorts $\left(I^{2}=61.9 \%, P=0.02\right)$. There was no evidence indicating significant publication bias (Begg's test, $P=0.14$; Egger's test, $P=0.57$ ).

\section{Circulating magnesium levels and hypertension risk}

Three studies (4 cohorts) reported data on incidence of hypertension (Fig. 3). The pooled RR of incident hypertension was 0.91 (95\% CI: $0.80,1.02 ; P=0.10$ ) comparing the highest to the lowest circulating $\mathrm{Mg}$ levels. No significant heterogeneity among studies was found $\left(I^{2}=0.0 \%, \quad P=0.48\right)$. Neither Egger's $(P=0.86)$ nor Begg's $(P=0.50)$ test indicated significant publication bias.

A significant inverse linear association was observed between circulating $\mathrm{Mg}$ levels and incidence of hypertension (RR, 0.96; 95\% CI: 0.93, 0.99; $P_{\text {trend }}=0.02$ with a $0.1 \mathrm{mmol} / \mathrm{L}$ increment in $\mathrm{Mg}$ levels). No significant heterogeneity across studies $\left(I^{2}=0.0 \%, P=0.69\right)$ and no evidence of publication bias were found (Egger's test: $P=0.62$; Begg's test: $P=0.50)$.

\section{Circulating magnesium levels and diabetic risk}

Four studies (5 cohorts) represented results on T2DM (Fig. 4). The pooled RR for incidence of T2DM comparing the highest to the lowest category of $\mathrm{Mg}$ levels was 0.64 (95\% CI: $0.50,0.81 ; P=0.01$ ). There was no significant heterogeneity among the studies $\left(I^{2}=27.3 \%\right.$, 


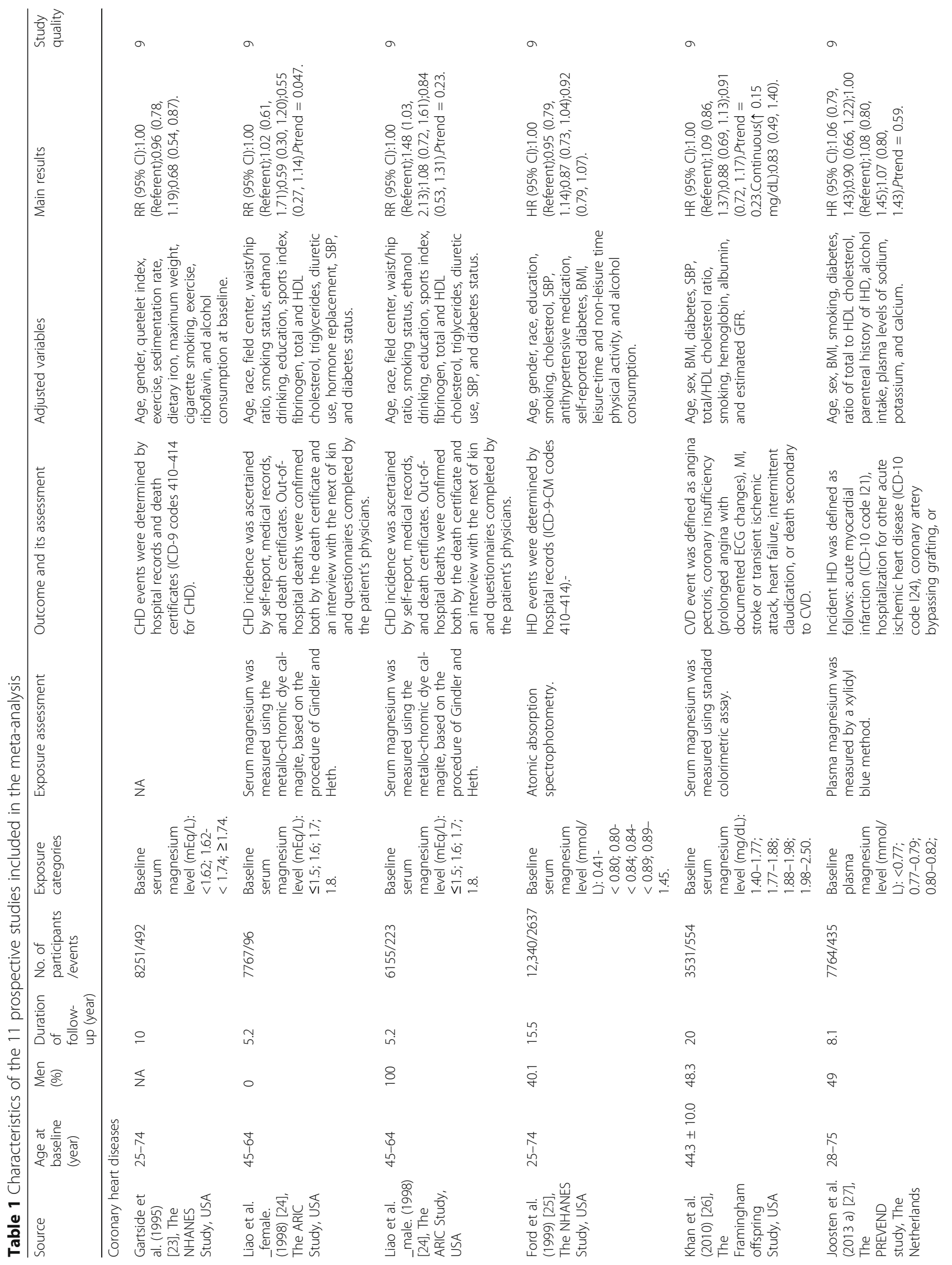




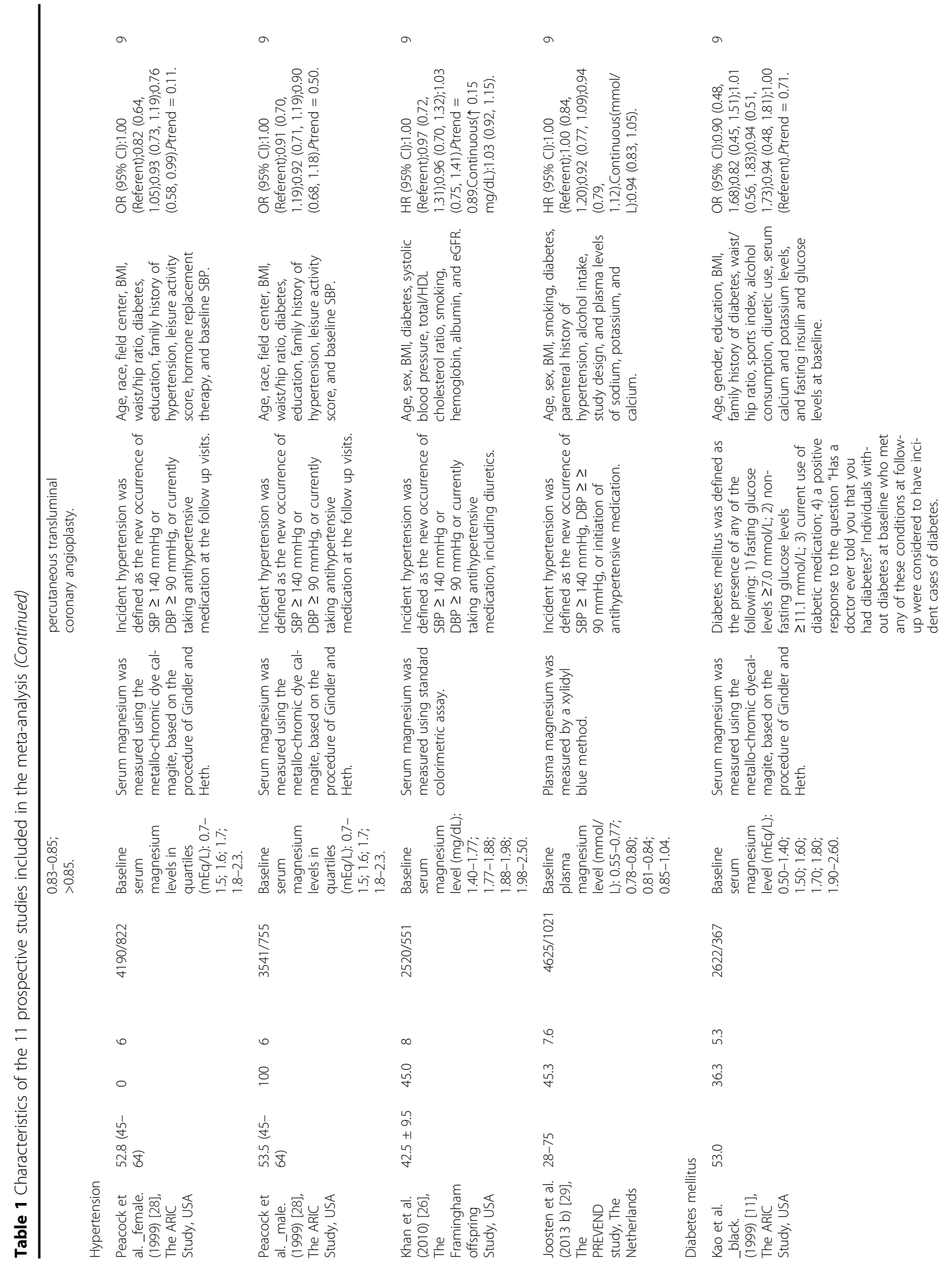




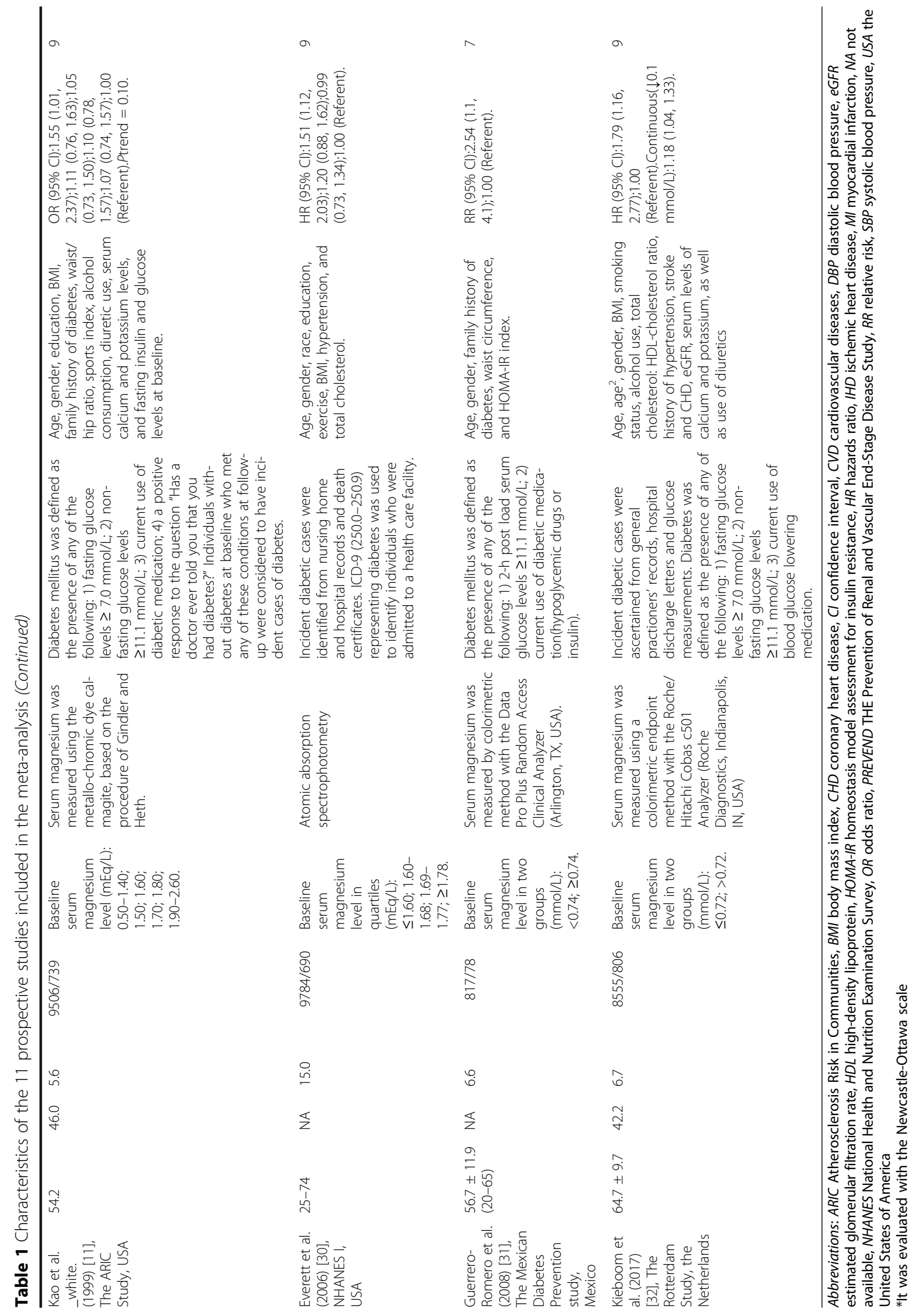




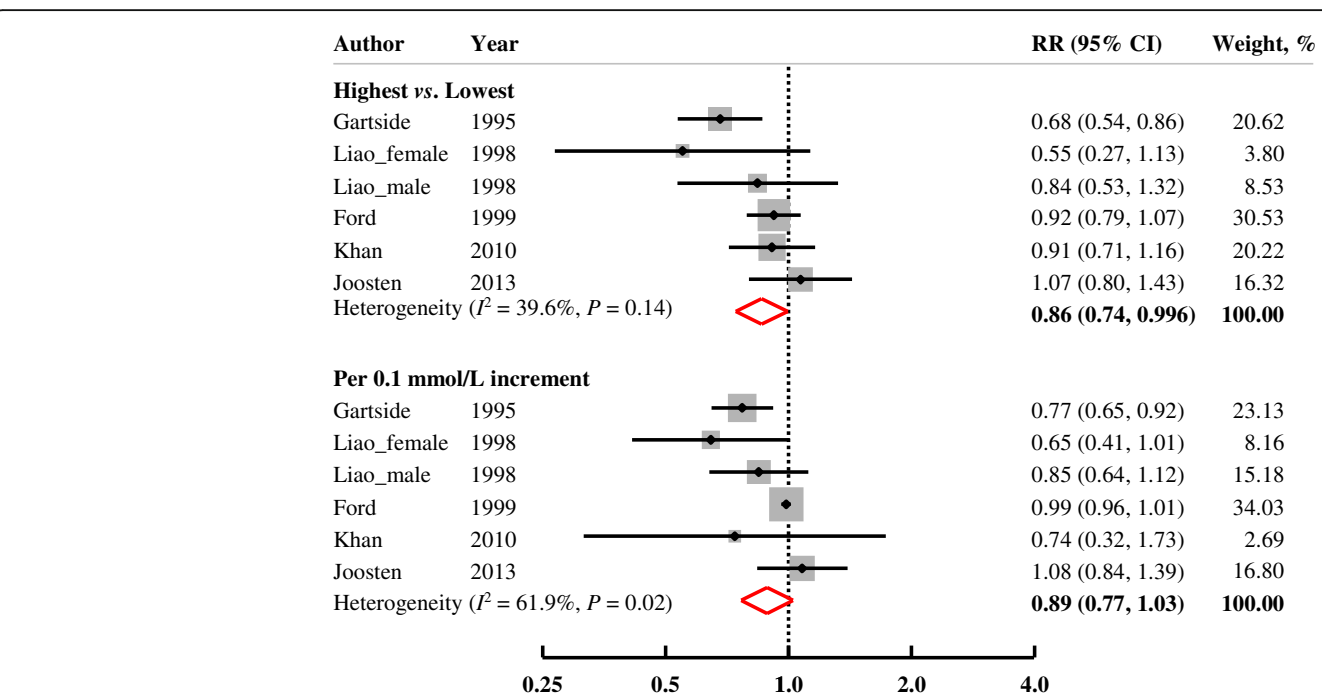

Fig. 2 Multivariable-adjusted RRs ( $95 \% \mathrm{Cls}$ ) for incidence of CHD comparing highest to lowest, or per $0.1 \mathrm{mmol} / \mathrm{L}$ increment in circulating Mg levels from prospective cohort studies. The summary estimate was obtained by using a random-effects model. The dots indicate the adjusted RRs. The size of the shaded square is proportional to the weight of each study. The horizontal lines represent 95\% Cls. The diamond markers indicate the pooled RRs. Abbreviations: CHD, coronary heart disease; Cl, confidence interval; RR, relative risk

$P=0.24)$, and no significant publication bias was found (Egger's test: $P=0.94$; Begg's test: $P=0.46$ ).

According to the available data from 3 cohorts, a nonsignificant linear association was observed (RR, 0.90; 95\% CI: $0.81,1.002 ; P_{\text {trend }}=0.054$ with a $0.1 \mathrm{mmol} / \mathrm{L}$ increment in circulating Mg levels). But, a high heterogeneity across studies was detected $\left(I^{2}=76.4 \%, P=0.01\right)$. No evidence of publication bias was found (Egger's test: $P=0.26$; Begg's test: $P=0.31$ ).
The funnel plots did not indicate publication bias for any pooling in this meta-analysis (See Additional file 1: Figure $\mathrm{S} 1$ in supplemental materials).

\section{Sensitivity analysis}

When replacing a random-effects model with a fixedeffects model, the results were generally consistent, except that the linear association between circulating $\mathrm{Mg}$ levels and incidence of T2DM became statistically significant

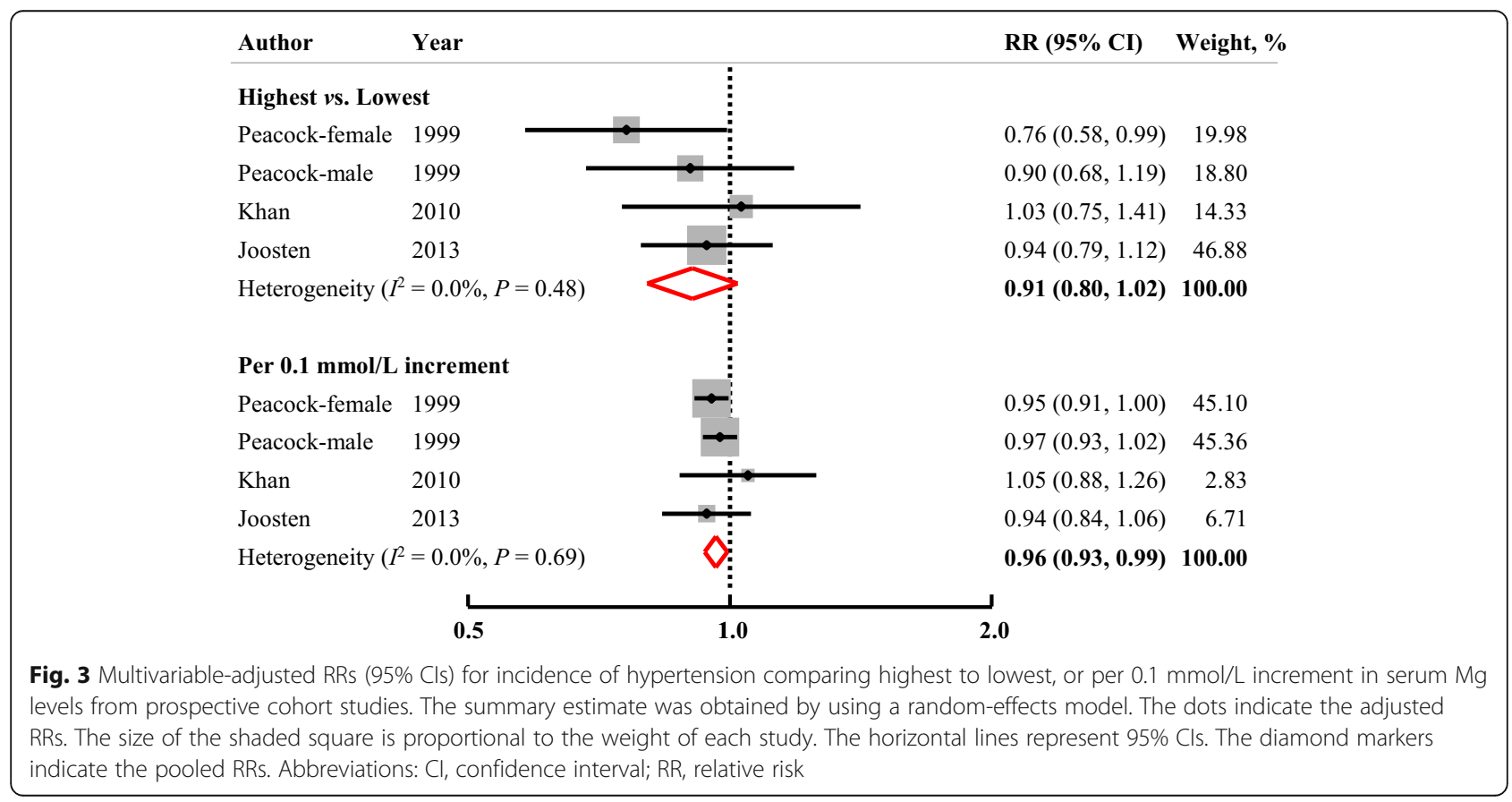




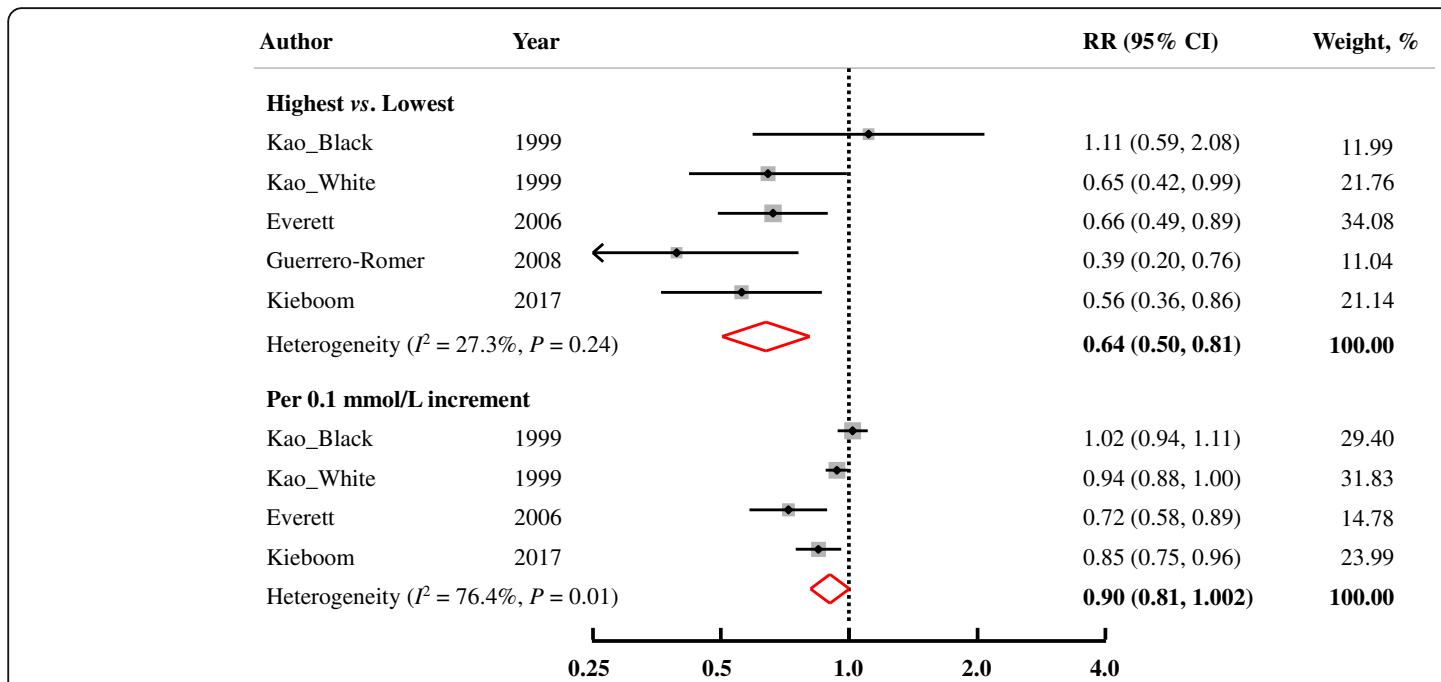

Fig. 4 Multivariable-adjusted RRs (95\% Cls) for incidence of T2DM comparing highest to lowest, or per $0.1 \mathrm{mmol} / \mathrm{L}$ increment in serum Mg levels from prospective cohort studies. The summary estimate was obtained by using a random-effects model. The dots indicate the adjusted RRs. The size of the shaded square is proportional to the weight of each study. The horizontal lines represent $95 \% \mathrm{Cls}$. The diamond markers indicate the pooled RRs. Abbreviations: Cl, confidence interval; RR, relative risk; T2DM, type 2 diabetes mellitus

(0.94; 95\% CI: 0.90, 0.98; $P<0.01$ ) (see Additional file 1: Table S3 in supplemental materials).

Additional file 1: Table S4 (see supplemental materials) presents the influence of a single study on the overall associations of interest by omitting one study at each time from the pooled analysis. The categorical association between circulating Mg levels (highest vs. lowest) and incidence of CHD was attenuated to some extent by omitting Gartside et al. [23] (RR, 0.92; 95\% CI: 0.82, 1.03; $P=0.16$ ), Liao et al. [24] (female cohort: RR, 0.88; 95\% CI: 0.76, 1.01; $P=0.07$; male cohort: RR, 0.86 ; $95 \%$ CI: $0.73,1.02$; $P=0.08$ ), Ford et al. [25] (RR, 0.83; 95\% CI: 0.68, 1.02; $P=0.08$ ), or Khan et al. [26] (RR, 0.84; 95\% CI: 0.70, 1.02; $P=0.08)$. The linear association between circulating $\mathrm{Mg}$ levels and incidence of CHD was strengthened and became statistically significant (RR, $0.84 ; 95 \%$ CI: $0.71,0.99$; $P=0.04$ ) when Ford et al. [25] was omitted.

No single study substantially changed the categorical association between circulating Mg levels (highest vs. lowest) and incidence of hypertension. However, when the female cohort from Peacock et al. [28] was omitted, the overall linear association between circulating Mg levels and incidence of hypertension was attenuated and became statistically non-significant (RR, $0.97 ; 95 \% \mathrm{CI}: 0.93,1.01 ; P=0.20$ ).

The overall categorical association between circulating Mg levels (highest vs. lowest) and incidence of T2DM persisted with excluding any single study each time in the meta-analysis, while the linear association between circulating $\mathrm{Mg}$ levels and incidence of T2DM was strengthened and became statistically significant when Kao et al. [11] (RR, 0.86; 95\% CI: 0.75, 0.97; $P=0.02$ ) was omitted in the meta-analysis.
In addition, when we transformed $O R$ to $R R$ in one study for hypertension [28], and the other one for diabetes [11], the related results were not materially changed (data not shown).

\section{Discussion}

Findings from this meta-analysis of prospective cohort studies suggested that circulating $\mathrm{Mg}$ levels were inversely associated with incidence of $\mathrm{CHD}$, hypertension and T2DM. The present study is complementary to the previous systematic reviews on dietary $\mathrm{Mg}$ intake and provides additional evidence in support of the potential beneficial effect of $\mathrm{Mg}$ on CVD and diabetes.

\section{Comparison with previous reviews}

Although no previous meta-analyses specifically focused on CHD, several meta-analyses have studied the association of circulating $\mathrm{Mg}$ levels and CVD risk [13, 34]. One meta-analysis of prospective studies published in 2013, which combined CVD incidence and mortality, reported that every $0.2 \mathrm{mmol} / \mathrm{L}$ increment in circulating $\mathrm{Mg}$ levels was associated with a $30 \%$ lower risk of CVD and a $17 \%$ lower risk of ischemic heart disease [13]. Another meta-analysis also published in 2013 found that the pooled RR of total CVD events (including CVD incidence and mortality) was $23 \%$ lower comparing the highest to the lowest serum Mg levels [34]. Also, a recent meta-analysis observed a significant but heterogeneous inverse association between serum $\mathrm{Mg}$ and metabolic syndrome [40]. Another recent meta-analysis investigated dietary $\mathrm{Mg}$ intake, but not circulating $\mathrm{Mg}$ level, in relation to type 2 diabetes and other CVD 
endpoints [41]. By contrast, we focused on research about CHD events and our findings contribute additional information to the literature.

To the best of our knowledge, this is the first systematic review to summarize studies on circulating $\mathrm{Mg}$ levels and incidence of hypertension. Our findings are generally consistent with previous reviews and metaanalyses of interventional studies on $\mathrm{Mg}$ supplementation [42-44]. For example, one meta-analysis of clinical trials found that $\mathrm{Mg}$ supplementation decreased systolic blood pressure (SBP) $(3-4 \mathrm{mmHg})$ and diastolic blood pressure (DBP) $(2-3 \mathrm{mmHg})$ in both normotensive and hypertensive individuals. [43] Another meta-analysis found that $\mathrm{Mg}$ supplementation significantly reduced SBP $(18.7 \mathrm{mmHg})$ and DBP $(10.9 \mathrm{mmHg})$ in hypertensive patients with SBP > $155 \mathrm{mmHg}$ [44].

Several meta-analyses examined the association of dietary $\mathrm{Mg}$ intake and diabetes risk [9, 45-47]. For example, an updated meta-analysis of prospective cohort studies published in 2015 found that Mg intake was significantly and inversely associated with risk of T2DM in a nonlinear dose-response manner $\left(P_{\text {nonlinearity }}=0.003\right)$ [45]. In addition, a most-recent published systematic review and meta-analysis of randomized clinical trials reported that oral $\mathrm{Mg}$ supplementation improved insulinsensitivity parameters in those who were at high risk for diabetes [48]. Similarly, findings from our meta-analysis of circulating $\mathrm{Mg}$ levels and risk of T2DM are supplementary to previous systematic reviews on dietary $\mathrm{Mg}$ intake or $\mathrm{Mg}$ supplementation.

\section{Strengths and limitations}

Our meta-analysis has several strengths. First, this is the first up-to-date meta-analysis examining the associations between circulating $\mathrm{Mg}$ levels and incidence of $\mathrm{CHD}$, hypertension, and T2DM together in the general population. Second, this meta-analysis was based on prospective cohort studies of high or moderate quality from various populations, which reduced the likelihood that our findings were substantially biased by the inherent limitations in the primary studies. Third, the combined sample size was relatively large and the long duration of follow-up enabled us to examine the long-term association. Considering that randomized controlled trials are relatively small and include short follow-up periods, our data provide important supplementary information to the literature. Finally, our conclusions are strengthened by generally consistent findings from both categorical and linear analyses, as well as the robust findings from the sensitivity analyses.

Some limitations also need to be acknowledged. Although we found significant associations, the present meta-analysis was based on 10 published cohort studies. The limited data sources not only restricted us from doing stratified or subgroup analyses, but also may potentially lead to over- or under-estimation of the true associations. Second, similar to other meta-analyses of observational studies, the inherent limitations in primary studies may bias the pooled results, though various potential confounders were considered in the original studies. Of note, factors such as family history of chronic diseases and medications related to $\mathrm{Mg}$ metabolism were seldom mentioned in the included studies. Third, the possibility of misclassification from both exposure and outcome could not be completely excluded. However, objective biomarkers were used for Mg assessment, and objective evidence (e.g., medical records and death certificates) was used for outcome assessment in most of the included studies. Fourth, although we did not observe evidence of publication bias, the likelihood could not be completely excluded due to publications in other languages. Finally, we realize that circulating $\mathrm{Mg}$ may not be the best biomarker, thus alternative biomarkers (e.g., red blood cell $\mathrm{Mg}$ and ionized $\mathrm{Mg}$ ) are definitely needed in future studies depending on the scale of the study and other considerations such as budget and ethics.

\section{Potential mechanisms}

$\mathrm{Mg}$ is a co-factor of more than 350 essential metabolic reactions. Most importantly, as a component of the Mgadenosine triphosphate complex, $\mathrm{Mg}$ is involved with all phosphate transfer reactions [6, 7, 49]. Experimental studies show that Mg could: 1) regulate vascular smooth muscle tone through modulating calcium entry and intracellular signal pathways [50,51]; and 2) regulate endothelial function through adjusting the synthesis and release of vasodilatory prostacyclin and nitric oxide [52, 53]. These vascular effects of $\mathrm{Mg}$ form the link between its deficiency and the pathogenesis of CHD and hypertension. Studies also found that Mg inhibited experimental arterial thrombus formation by inhibition of platelet aggregation [54], and $\mathrm{Mg}$ deficiency resulted in inflammation in various parts of the heart [55] and acceleration of the atherosclerotic process [56], which were related to the development of CHD. In recent metaanalyses and reviews, researchers found that low diet $\mathrm{Mg}$ intake and hypomagnesemia were correlated with lowgrade inflammation and oxidative stress [57-59], both of which are known to be part of the pathogenesis of chronic diseases such as CHD and T2DM.

In addition to $\mathrm{Mg}$ involvement in glucose metabolism, including the glycolytic pathway and the Krebs cycle, studies have shown that $\mathrm{Mg}$ is essential in insulin signal transduction through the activation of the $\beta$-subunit of the tyrosine kinase domain of the insulin receptor, which is a critical step in the trans-membrane signaling cascade of the insulin reaction $[6,7,60]$. Animal experiments 
found that Mg deficiency was associated with reduced glucose uptake and utilization in insulin-sensitive tissues, thus promoting insulin resistance and the development of diabetes [25, 61]. Furthermore, randomized controlled trials including non-diabetic individuals found improvement in insulin sensitivity together with increased levels of serum $\mathrm{Mg}$ after $\mathrm{Mg}$ supplementation [62-64].

\section{Conclusions}

In conclusion, this meta-analysis of prospective cohort studies found that circulating $\mathrm{Mg}$ was inversely associated with incidence of CHD, hypertension, and T2DM. Findings of this meta-analysis are supplementary to previous reviews on dietary $\mathrm{Mg}$ intake and risk of CVD and diabetes. Further studies are needed to provide more solid evidence, and to elucidate the dose-response relationship and to explore the optimal range of circulating $\mathrm{Mg}$ concentrations in terms of prevention of CHD, hypertension, and T2DM.

\section{Additional file}

Additional file 1: Table S1. PRISMA checklist. Table S2. Quality assessment using Newcastle-Ottawa quality assessment scale for the studies included in the meta-analysis. Table S3. Multivariable adjusted association of circulating magnesium levels with incidence of $\mathrm{CHD}$, hypertension, and T2DM using a fixed-effects model: a sensitivity analysis. Table S4. Influence of a single study on the pooled association of circulating magnesium levels with incidence of CHD, hypertension, and T2DM using a random-effects model: a sensitivity analysis. Figure S1. Funnel plots with pseudo $95 \%$ CLs for six pooling in this meta-analysis. (DOC $202 \mathrm{~kb}$ )

\section{Abbreviations}

CHD: Coronary heart diseases; Cl: Confidence interval; CVD: Cardiovascular diseases; DBP: Diastolic blood pressure; HR: Hazard ratio; Mg: Magnesium; NOS: The Newcastle-Ottawa quality assessment scale; OR: Odds ratio; PRISMA: The preferred reporting items for systematic reviews and metaanalyses; RR: Relative risk; SBP: Systolic blood pressure; T2DM: Type 2 diabetes

\section{Acknowledgments}

Not applicable

\section{Funding}

This study was supported by one grant from Shanghai Key Laboratory of Pediatric Gastroenterology and Nutrition (14DZ2272400, to WC), China and one grant from the National Institute of Health, USA (R01ES021735, to KH).

\section{Availability of data and materials}

All data generated or analyzed during this study are included in these published articles [11, 23-32]. There are no additional data.

\section{Authors' contributions}

WC: administrative support; KH and WC: study concept and design; JW PX and QT: literature search, study selection, and data extraction; PX: statistical analyses; JW and PX: preparing the tables and figures; JW, PX and $\mathrm{KH}$ : drafting the original manuscript; $\mathrm{WC}$ and $\mathrm{KH}$ : supervision of all the work. All the authors completely consented with all the data in the study, critically reviewed the manuscript for important intellectual content, and approved the final manuscript. WC and KH had primary responsibility for the final manuscript.

Ethics approval and consent to participate Not applicable.
Consent for publication

Not applicable.

\section{Competing interests}

The authors declare that they have no competing interests.

\section{Publisher's Note}

Springer Nature remains neutral with regard to jurisdictional claims in published maps and institutional affiliations.

\section{Author details}

'Department of Clinical Nutrition, Xin Hua Hospital, School of Medicine, Shanghai Jiao Tong University, No. 1665, Kongjiang Rd, Shanghai, China. ${ }^{2}$ Shanghai Key Laboratory of Pediatric Gastroenterology and Nutrition, Shanghai, China. ${ }^{3}$ Shanghai Institute for Pediatric Research, Shanghai, China. ${ }^{4}$ Department of Epidemiology and Biostatistics, School of Public Health-Bloomington, Indiana University, 1025 E. Seventh Street, C042, Bloomington, Indiana 47405, USA. ${ }^{5}$ Department of Pediatric Surgery, Xin Hua Hospital, School of Medicine, Shanghai Jiao Tong University, Shanghai, China.

Received: 10 January 2017 Accepted: 24 July 2017

Published online: 19 September 2017

\section{References}

1. Gress TW, Nieto FJ, Shahar E, Wofford MR, Brancati FL. Hypertension and antihypertensive therapy as risk factors for type 2 diabetes mellitus. Atherosclerosis risk in communities study. N Engl J Med. 2000;342:905-12.

2. Ma J, Folsom AR, Melnick SL, Eckfeldt JH, Sharrett AR, Nabulsi AA, Hutchinson RG, Metcalf PA. Associations of serum and dietary magnesium with cardiovascular disease, hypertension, diabetes, insulin, and carotid arterial wall thickness: the ARIC study. Atherosclerosis risk in communities study. J Clin Epidemiol. 1995;48:927-40.

3. Guariguata L, Whiting DR, Hambleton I, Beagley J, Linnenkamp U, Shaw JE. Global estimates of diabetes prevalence for 2013 and projections for 2035. Diabetes Res Clin Pract. 2014;103:137-49.

4. Danaei G, Finucane MM, Lu Y, Singh GM, Cowan MJ, Paciorek CJ, Lin JK, Farzadfar F, Khang YH, Stevens GA, et al. National, regional, and global trends in fasting plasma glucose and diabetes prevalence since 1980: systematic analysis of health examination surveys and epidemiological studies with 370 country-years and 2.7 Million participants. Lancet. 2011;378:31-40.

5. Liang Y, Liu R, Du S, Qiu C. Trends in incidence of hypertension in Chinese adults, 1991-2009: the China health and nutrition survey. Int J Cardiol. 2014; 175:96-101.

6. Takaya J, Higashino H, Kobayashi Y. Intracellular magnesium and insulin resistance. Magnes Res. 2004;17:126-36.

7. Gunther T. The biochemical function of $\mathrm{mg}(2)+$ in insulin secretion, insulin signal transduction and insulin resistance. Magnes Res. 2010;23:5-18.

8. Champagne CM. Magnesium in hypertension, cardiovascular disease, metabolic syndrome, and other conditions: a review. Nutr Clin Pract. 2008; 23:142-51.

9. Dong JY, Xun P, He K, Qin LQ. Magnesium intake and risk of type 2 diabetes: meta-analysis of prospective cohort studies. Diabetes Care. 2011; 34:2116-22.

10. Kaur B, Henry J. Micronutrient status in type 2 diabetes: a review. Adv Food Nutr Res. 2014;71:55-100.

11. Kao WH, Folsom AR, Nieto FJ, Mo JP, Watson RL, Brancati FL. Serum and dietary magnesium and the risk for type 2 diabetes mellitus: the atherosclerosis risk in communities study. Arch Intern Med. 1999:159:2151-9.

12. Kim DJ, Xun P, Liu K, Loria C, Yokota K, Jacobs DR Jr, He K. Magnesium intake in relation to systemic inflammation, insulin resistance, and the incidence of diabetes. Diabetes Care. 2010;33:2604-10.

13. Del Gobbo LC, Imamura F, Wu JH, de Oliveira Otto MC, Chiuve SE, Mozaffarian D. Circulating and dietary magnesium and risk of cardiovascular disease: a systematic review and meta-analysis of prospective studies. Am J Clin Nutr. 2013;98:160-73.

14. Lopez-Ridaura R, Willett WC, Rimm EB, Liu S, Stampfer MJ, Manson JE, Hu FB. Magnesium intake and risk of type 2 diabetes in men and women. Diabetes Care. 2004;27:134-40.

15. Al-Delaimy WK, Rimm EB, Willett WC, Stampfer MJ, Hu FB. Magnesium intake and risk of coronary heart disease among men. J Am Coll Nutr. 2004;23:63-70. 
16. Song Y, Sesso HD, Manson JE, Cook NR, Buring JE, Liu S. Dietary magnesium intake and risk of incident hypertension among middle-aged and older US women in a 10-year follow-up study. Am J Cardiol. 2006;98:1616-21.

17. Kimura M, Itokawa Y. Cooking losses of minerals in foods and its nutritional significance. J Nutr Sci Vitaminol (Tokyo). 1990;36(Suppl 1):S25-32. discussion $\mathrm{S33}$

18. Elin RJ. Assessment of magnesium status for diagnosis and therapy. Magnes Res. 2010;23:S194-8.

19. Zhang X, Del Gobbo LC, Hruby A, Rosanoff A, He K, Dai Q, Costello RB, Zhang W, Song Y. The circulating concentration and 24-h urine excretion of magnesium dose- and time-dependently respond to oral magnesium supplementation in a meta-analysis of randomized controlled trials. J Nutr. 2016;146:595-602.

20. Paolisso G, Scheen A, D'Onofrio F, Lefebvre P. Magnesium and glucose homeostasis. Diabetologia. 1990;33:511-4.

21. Simmons $D$, Joshi $S$, Shaw J. Hypomagnesaemia is associated with diabetes: not pre-diabetes, obesity or the metabolic syndrome. Diabetes Res Clin Pract. 2010;87:261-6.

22. Xun $P, W u Y, H e ~ Q, ~ H e ~ K$. Fasting insulin concentrations and incidence of hypertension, stroke, and coronary heart disease: a meta-analysis of prospective cohort studies. Am J Clin Nutr. 2013;98:1543-54.

23. Gartside PS, Glueck CJ. The important role of modifiable dietary and behavioral characteristics in the causation and prevention of coronary heart disease hospitalization and mortality: the prospective NHANES I follow-up study. J Am Coll Nutr. 1995;14:71-9.

24. Liao F, Folsom AR, Brancati FL. Is low magnesium concentration a risk factor for coronary heart disease? The atherosclerosis risk in communities (ARIC) study. Am Heart J. 1998;136:480-90.

25. Ford ES. Serum magnesium and ischaemic heart disease: findings from a national sample of US adults. Int J Epidemiol. 1999;28:645-51.

26. Khan AM, Sullivan L, McCabe E, Levy D, Vasan RS, Wang TJ. Lack of association between serum magnesium and the risks of hypertension and cardiovascular disease. Am Heart J. 2010;160:715-20.

27. Joosten MM, Gansevoort RT, Mukamal KJ, van der Harst P, Geleijnse JM, Feskens EJ, Navis G, Bakker SJ, Group PS. Urinary and plasma magnesium and risk of ischemic heart disease. Am J Clin Nutr. 2013:97:1299-306.

28. Peacock JM, Folsom AR, Arnett DK, Eckfeldt JH, Szklo M. Relationship of serum and dietary magnesium to incident hypertension: the atherosclerosis risk in communities (ARIC) study. Ann Epidemiol. 1999;9:159-65.

29. Joosten MM, Gansevoort RT, Mukamal KJ, Kootstra-Ros JE, Feskens EJ, Geleijnse JM, Navis G, Bakker SJ, Group PS. Urinary magnesium excretion and risk of hypertension: the prevention of renal and vascular end-stage disease study. Hypertension. 2013;61:1161-7.

30. Everett CJ, King DE. Serum magnesium and the development of diabetes. Nutrition. 2006:22:679.

31. Guerrero-Romero F, Rascon-Pacheco RA, Rodriguez-Moran M, de la Pena JE, Wacher N. Hypomagnesaemia and risk for metabolic glucose disorders: a 10-year follow-up study. Eur J Clin Investig. 2008;38:389-96.

32. Kieboom BC, Ligthart S, Dehghan A, Kurstjens S, de Baaij JH, Franco OH, Hofman A, Zietse R, Stricker BH, Hoorn EJ. Serum magnesium and the risk of prediabetes: a population-based cohort study. Diabetologia. 2017;60:843-53.

33. Han H, Fang X, Wei X, Liu Y, Jin Z, Chen Q, Fan Z, Aaseth J, Hiyoshi A, He J, Cao Y. Dose-response relationship between dietary magnesium intake, serum magnesium concentration and risk of hypertension: a systematic review and meta-analysis of prospective cohort studies. Nutr J. 2017;16:26.

34. Qu X, Jin F, Hao Y, Li H, Tang T, Wang H, Yan W, Dai K. Magnesium and the risk of cardiovascular events: a meta-analysis of prospective cohort studies. PLoS One. 2013;8:e57720.

35. Moher D, Liberati A, Tetzlaff J, Altman DG, Group P. Preferred reporting items for systematic reviews and meta-analyses: the PRISMA statement. J Clin Epidemiol. 2009:62:1006-12.

36. The Newcastle-Ottawa Scale (NOS) for assessing the quality of nonrandomised studies in meta-analyses. http://www.ohri.ca/programs/ clinical_epidemiology/oxford.asp. Accessed 20 Dec 2016.

37. Zhang J, Yu KF. What's The relative risk? A method of correcting the odds ratio in cohort studies of common outcomes. JAMA. 1998;280:1690-1.

38. Greenland S, Longnecker MP. Methods for trend estimation from summarized dose-response data, with applications to meta-analysis. Am J Epidemiol. 1992;135:1301-9.

39. Duval S, Tweedie R. A nonparametric "trim and fill" method of accounting for publication bias in meta-analysis. J Am Stat Assoc. 2000;95:89-98.
40. Sarrafzadegan N, Khosravi-Boroujeni H, Lotfizadeh M, Pourmogaddas A, Salehi-Abargouei A. Magnesium status and the metabolic syndrome: a systematic review and meta-analysis. Nutrition. 2016;32:409-17.

41. Fang X, Wang K, Han D, He X, Wei J, Zhao L, Imam MU, Ping Z, Li Y, Xu Y, et al. Dietary magnesium intake and the risk of cardiovascular disease, type 2 diabetes, and all-cause mortality: a dose-response meta-analysis of prospective cohort studies. BMC Med. 2016;14:210.

42. Jee SH, Miller ER 3rd, Guallar E, Singh VK, Appel LJ, Klag MJ. The effect of magnesium supplementation on blood pressure: a meta-analysis of randomized clinical trials. Am J Hypertens. 2002;15:691-6.

43. Kass L, Weekes J, Carpenter L. Effect of magnesium supplementation on blood pressure: a meta-analysis. Eur J Clin Nutr. 2012;66:411-8.

44. Rosanoff A, Plesset MR. Oral magnesium supplements decrease high blood pressure (SBP>155 $\mathrm{mmHg}$ ) in hypertensive subjects on anti-hypertensive medications: a targeted meta-analysis. Magnes Res. 2013;26:93-9.

45. Xu T, Chen GC, Zhai L, Ke KF. Nonlinear reduction in risk for type 2 diabetes by magnesium intake: an updated meta-analysis of prospective cohort studies. Biomed Environ Sci. 2015;28:527-34.

46. Larsson SC, Wolk A. Magnesium intake and risk of type 2 diabetes: a metaanalysis. J Intern Med. 2007;262:208-14.

47. Schulze MB, Schulz M, Heidemann C, Schienkiewitz A, Hoffmann K, Boeing $H$. Fiber and magnesium intake and incidence of type 2 diabetes: a prospective study and meta-analysis. Arch Intern Med. 2007;167:956-65.

48. Veronese N, Watutantrige-Fernando S, Luchini C, Solmi M, Sartore G, Sergi G, Manzato E, Barbagallo M, Maggi S, Stubbs B. Effect of magnesium supplementation on glucose metabolism in people with or at risk of diabetes: a systematic review and meta-analysis of double-blind randomized controlled trials. Eur J Clin Nutr. 2016;70:1354-9.

49. Sales CH, Pedrosa LF. Magnesium and diabetes mellitus: their relation. Clin Nutr. 2006;25:554-62.

50. Altura BM, Altura BT. Magnesium, electrolyte transport and coronary vascular tone. Drugs. 1984;28(Suppl 1):120-42.

51. Mubagwa K, Gwanyanya A, Zakharov S, Macianskiene R. Regulation of cation channels in cardiac and smooth muscle cells by intracellular magnesium. Arch Biochem Biophys. 2007;458:73-89.

52. Maier JA, Malpuech-Brugere C, Zimowska W, Rayssiguier Y, Mazur A. Low magnesium promotes endothelial cell dysfunction: implications for atherosclerosis, inflammation and thrombosis. Biochim Biophys Acta. 1689:2004:13-21.

53. Pearson PJ, Evora PR, Seccombe JF, Schaff HV. Hypomagnesemia inhibits nitric oxide release from coronary endothelium: protective role of magnesium infusion after cardiac operations. Ann Thorac Surg. 1998; 65:967-72.

54. Ravn HB, Kristensen SD, Vissinger H, Husted SE. Magnesium inhibits human platelets. Blood Coagul Fibrinolysis. 1996;7:241-4.

55. Shah NC, Shah GJ, Li Z, Jiang XC, Altura BT, Altura BM. Short-term magnesium deficiency downregulates telomerase, upregulates neutral sphingomyelinase and induces oxidative DNA damage in cardiovascular tissues: relevance to atherogenesis, cardiovascular diseases and aging. Int J Clin Exp Med. 2014;7:497-514.

56. Altura BT, Brust M, Bloom S, Barbour RL, Stempak JG, Altura BM. Magnesium dietary intake modulates blood lipid levels and atherogenesis. Proc Natl Acad Sci U S A. 1990:87:1840-4.

57. Nielsen FH. Effects of magnesium depletion on inflammation in chronic disease. Curr Opin Clin Nutr Metab Care. 2014;17:525-30.

58. Dibaba DT, Xun P, He K. Dietary magnesium intake is inversely associated with serum C-reactive protein levels: meta-analysis and systematic review. Eur J Clin Nutr. 2014;68:510-6.

59. Zheltova AA, Kharitonova MV, lezhitsa IN, Spasov AA. Magnesium deficiency and oxidative stress: an update. Biomedicine (Taipei). 2016;6:20.

60. Crill CM, Christensen ML, Storm MC, Helms RA. Relative bioavailability of carnitine supplementation in premature neonates. JPEN J Parenter Enteral Nutr. 2006;30:421-5

61. Balon TW, Gu JL, Tokuyama Y, Jasman AP, Nadler JL. Magnesium supplementation reduces development of diabetes in a rat model of spontaneous NIDDM. Am J Phys. 1995;269:E745-52.

62. Christ F, Raithel P, Gartside IB, Gamble J, Peter K, Messmer K. Investigating the origin of cyclic changes in limb volume using mercury-in-silastic strain gauge plethysmography in man. J Physiol. 1995;487(Pt 1):259-72.

63. Simental-Mendia LE, Rodriguez-Moran M, Guerrero-Romero F. Oral magnesium supplementation decreases C-reactive protein levels in subjects 
with prediabetes and hypomagnesemia: a clinical randomized double-blind placebo-controlled trial. Arch Med Res. 2014;45:325-30.

64. Rodriguez-Moran M, Guerrero-Romero F. Oral magnesium supplementation improves the metabolic profile of metabolically obese, normal-weight individuals: a randomized double-blind placebo-controlled trial. Arch Med Res. 2014:45:388-93.

Submit your next manuscript to BioMed Central and we will help you at every step:

- We accept pre-submission inquiries

- Our selector tool helps you to find the most relevant journal

- We provide round the clock customer support

- Convenient online submission

- Thorough peer review

- Inclusion in PubMed and all major indexing services

- Maximum visibility for your research

Submit your manuscript at www.biomedcentral.com/submit 